\title{
WGEMLab-A Simulation Platform for the Analysis of Rectangular Waveguide Aperture Radiation
}

\author{
Ioan E. Lager, Cristian I. Coman, and Adrianus T. de Hoop
}

\begin{abstract}
A simulation platform for the analysis of the aperture radiation from a (dielectric-filled) rectangular waveguide is described. It consists of two computational engines and a userfriendly shell for controlling the configurational parameters and for selecting the postprocessing tasks. The platform is extremely suited for educational purposes.
\end{abstract}

Index Terms-Computer-aided instruction, electromagnetic analysis, mode-matching methods.

\section{INTRODUCTION}

$\mathbf{T}$ HE USE of (dielectric-filled) waveguide apertures as radiating elements is a topic that occurs in most courses on antenna theory and design. The study of this topic demands a lot of insight in a variety of extremely complex phenomena, such as propagation of electromagnetic perturbations through inhomogeneous configurations and in the free space or the singular behavior of the electromagnetic field near sharp edges. The mathematical description of these phenomena, while ensuring the accuracy and the rigor of the presentation of the topic, has a highly abstract character. Consequently, it often tends to obscure the physical meaning of otherwise extremely intuitive aspects. Didactic experience demonstrates that the needed insight is acquired easier when the theoretical exposition is complemented by hands-on experiments. However, in the case of antenna courses, physical experiments require complex and costly setups, rendering this choice infeasible.

It then follows that computer simulations present the adequate alternative for the familiarization of the student with the relevant phenomena. It is, however, noted that for meeting the specific requirements of the educational process, the employed software tools should provide the computed results in an extremely intuitive manner. In this respect, the graphical postprocessing capabilities are of paramount importance. Further, animation can play an important role in the illustration of time dependent phenomena. Finally, the software should ensure the possibility to observe in "real time" how the modification of the design parameters of the investigated configurations influences the propagation and radiation properties [1].

To address these challenges, a number of modeling tools for the analysis of dielectric-filled flanged rectangular waveguide

Manuscript received June 18, 2002.

I. E. Lager and C. I. Coman are with the International Research Centre for Telecommunications-Transmission and Radar, Faculty of Information Technology and Systems, Delft University of Technology, 2628 CD Delft, The Netherlands (e-mail: I.Lager@ITS.TUDelft.NL; C.Coman@IRCTR.TUDelft. NL).

A. T. de Hoop is with the Laboratory for Electromagnetic Research, Faculty of Information Technology and Systems, Delft University of Technology, 2628 CD Delft, The Netherlands (e-mail: A.T.deHoop@ITS.TUDelft.NL).

Digital Object Identifier 10.1109/TMAG.2003.810166

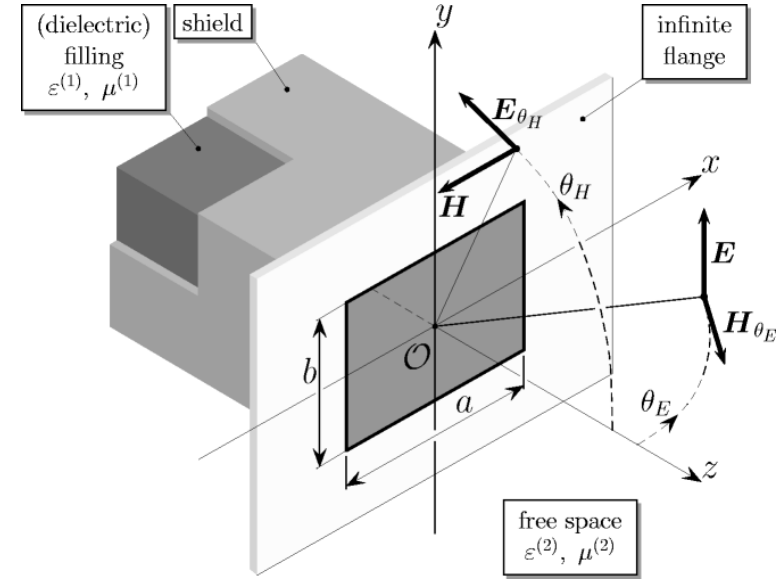

Fig. 1. Flanged rectangular waveguide antenna.

aperture radiation were developed recently at the International Research Centre for Telecommunications-Transmission and Radar (IRCTR). These tools were combined with a user-friendly shell, resulting in a simulation platform for educational purposes, called WGEMLab. For ensuring the accuracy and the computational speed required in an education environment, the platform employs the mode-matching computational technique [2]. Most of the modules included in the present version of WGEMLab are implemented in Matlab. However, for computational efficiency considerations, the employed computational engines are partially implemented in $\mathrm{C}^{++}$.

The first part of our analysis is concerned with the description of the WGEMLab platform's overall structure. The employed computational technique, as well as some implementation aspects, will be presented. In the second part, the potential of the package in the educational process will be demonstrated by examining some case studies.

\section{The Structure of the WGEMLab Platform}

\section{A. Analyzed Configuration}

The analyzed configuration concerns the radiation from the aperture of a (dielectric-filled) flanged rectangular waveguide (see Fig. 1). The subdomain in the interior of the waveguide $\{-a / 2<x<a / 2,-b / 2<y<b / 2,-\infty<z<0\}$ (with $a$ and $b$ denoting the width and the height of the waveguide, respectively) is filled with a homogeneous, isotropic, lossless medium with permittivity $\varepsilon^{(1)}$ and permeability $\mu^{(1)}$. The waveguide radiates into the half-space $\{-\infty<x<\infty,-\infty<y<$ $\infty, 0<z<\infty\}$, where the medium is taken to be homogeneous and isotropic and characterized by the permittivity $\varepsilon^{(2)}$ and the permeability $\mu^{(2)}$. The perfectly conductive flange extends to infinity. At any location in the investigated configura- 
tion, the electromagnetic field's state is described, in the time Laplace domain, by the electric field strength $\hat{E}(\boldsymbol{r}, j \omega)$ and by the magnetic field strength $\hat{\boldsymbol{H}}(\boldsymbol{r}, j \omega)$ (with $\boldsymbol{r}=x \boldsymbol{i}_{x}+y \boldsymbol{i}_{y}+z \boldsymbol{i}_{z}$ denoting the position vector, $j=\sqrt{-1}$ and $\omega \geqslant 0$ denoting the angular frequency). The rectangular waveguide is excited by means of a $\mathrm{TE}_{10}$ - mode of unit amplitude.

\section{B. Employed Computational Technique}

A key aspect concerning the radiation from waveguide apertures is the efficient and accurate modeling of the field behavior in the vicinity of the aperture. This aspect becomes even more critical when, in view of reducing the physical size of the aperture, the permittivity of the filling of the waveguide is taken to be very high [3]. For modeling the field behavior, both numerical techniques (for example, the finite-difference (FD) or finite-element (FE) methods) and (semi-)analytic ones were proposed.

Standard numerical techniques, such as FD or FE, allow the investigation of extremely complex configurations. Nevertheless, the accuracy of the computed results depends on the employed discretization. Furthermore, these results are sensitive to the (artificial) absorbing boundary conditions employed at the boundary of the domain of computation, that is necessarily of bounded support. These aspects conflict with the demands for flexibility, accuracy and, most of all, computational speed raised by the educational process. It then follows that other techniques (preferably, devoid of any kind of mesh) that suit better the highly regular character of the investigated configuration should be called upon.

Based on the arguments above, the computational engines of the WGEMLab simulation platform implement different versions of the (semi-analytic) mode-matching technique. It should be noted that, standardly, this method is applied to guided fields [2]. For tackling the examined electromagnetic problem, in which the radiation into the free space plays a key role, the following choices were made.

- The modal expansion of the field in the interior of the waveguide is matched to a spatial Fourier representation of the field in free space [4] (hereafter referred to as the MM-F technique).

- The modal expansion of the field in the interior of the waveguide is matched to a Green's function representation of the field in free space [3] (hereafter referred to as the MM-G technique).

The following options are available in the current version of the WGEMLab software package.

1) The two-dimensional (2-D) analysis of two parallel-plate waveguides of widths $a$ and $b$ (see Fig. 1), corresponding to the $E$ and $H$ polarizations, respectively. This analysis is representative for the behavior of the electromagnetic field along the waveguide's symmetry planes $\{y=0\}$ and $\{x=0\}$, respectively. The field quantities that are of relevance in this case are $\hat{E}_{y}\left(\boldsymbol{r}^{\prime}, j \omega\right), \hat{H}_{x}\left(\boldsymbol{r}^{\prime}, j \omega\right)$ and $\hat{H}_{z}\left(\boldsymbol{r}^{\prime}, j \omega\right)$ (with $\boldsymbol{r}^{\prime}=x \boldsymbol{i}_{x}+z \boldsymbol{i}_{z}$ ) for $E$ polarization and $\hat{E}_{y}\left(\boldsymbol{r}^{\prime \prime}, j \omega\right), \hat{E}_{z}\left(\boldsymbol{r}^{\prime \prime}, j \omega\right)$ and $\hat{H}_{x}\left(\boldsymbol{r}^{\prime \prime}, j \omega\right)$ (with $\boldsymbol{r}^{\prime \prime}=$ $\left.y \boldsymbol{i}_{y}+z \boldsymbol{i}_{z}\right)$ for $H$ polarization. The MM-F technique is employed.

2) The full three-dimensional (3-D) analysis of a rectangular waveguide. The MM-G technique is employed.

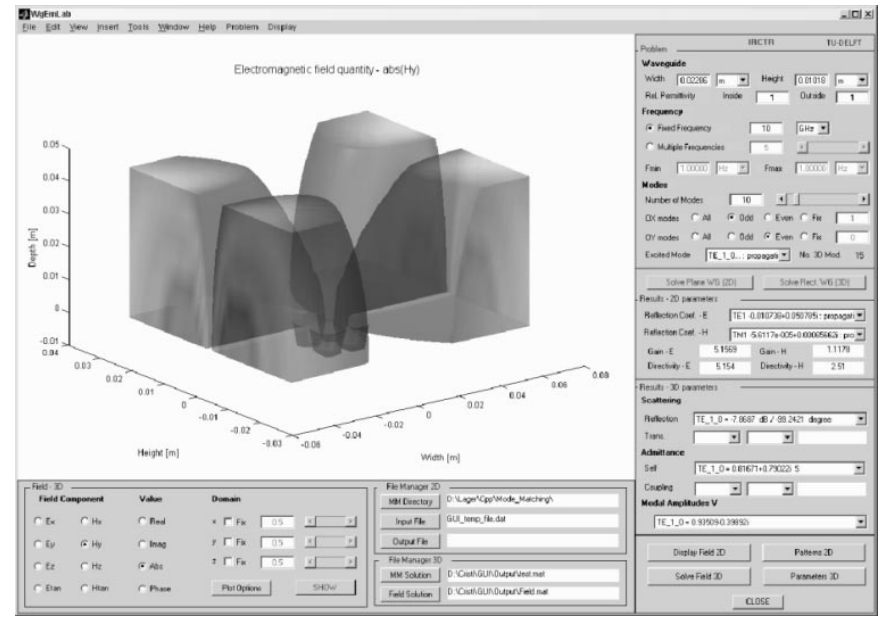

Fig. 2. The user interface.

\section{The Architecture of the WGEMLab Simulation Platform}

The WGEMLab simulation platform consists of two separate computational engines that perform the 2-D and 3-D analyses mentioned above. Each computational engine is complemented by an extended set of (graphic) postprocessing options. The options implemented in the current version of the WGEMLab simulation platform include the following.

\section{2-D Analysis (The MM-F Computational Engine):}

- computation of typical antenna parameters (gain, directivity, radiation pattern);

- surface/filled contour representations of the field inside the waveguide and in the near-field region;

- animated surface/filled contour representations of the field inside the waveguide and in the near-field region;

- spectral diagram of the antenna's radiation properties over a user-specified frequency range;

\section{3-D Analysis (The MM-G Computational Engine):}

- computation of the mutual coupling between the computed modes [3];

- computation of typical (circuital) antenna parameters;

- volume representations of the field inside the waveguide and in the near-field region; the representations include iso-surfaces and can make use of transparency; the value of the field quantities can be inspected along user-specified surfaces and lines and at user-specified locations.

The two computational engines are merged under a common graphic user interface (GUI) (see Fig. 2) that facilitates the setting up of the investigated configurations, the control of the design and functional parameters, and the selection of (graphic) postprocessing tasks.

The package, as a whole, is designed to run under the Matlab environment. However, for reducing the run times, the MM-F computational engine is delivered as a directly executable program (implemented in $\mathrm{C}^{++}$) that calls the Matlab engine, only, for graphic postprocessing purposes. Consequently, the MM-F engine can also be employed as a standalone application. 


\section{SUGGestions FOR THE ClASSROOM}

The possibilities of using the WGEMLab simulation platform in the educational process are demonstrated by examining some case studies. Note that throughout these analyses, dimensions will be given in meters.

\section{A. Proposed Case Studies}

The following field problems are proposed.

1) The 2-D analysis of two parallel-plate empty waveguides of widths $0.02 \mathrm{~m}$, corresponding to the $E$ and $H$ polarizations, respectively. The results are computed for:

- a fixed frequency $f=7.5098 \mathrm{GHz}$ (marginally exceeding the cutoff frequency of the first $E$ polarized, propagating mode); the field quantities are computed using 50 modes for both the $E$ and the $H$ polarization;

- a frequency range $f=7.51, \ldots, 40 \mathrm{GHz}$, sampled at 800 equidistant frequencies; the field quantities are computed using 10 modes for both the $E$ and the $H$ polarization.

2) The 3-D analysis of a WR90 rectangular waveguide of dimensions $a=0.02286 \mathrm{~m}$ and $b=0.01016 \mathrm{~m}$. The results are computed for a fixed frequency $f=10.5 \mathrm{GHz}$ and the field quantities are computed using $24 T E$ and $T M$ modes.

3) The 3-D spectral analysis of a WR90 rectangular waveguide and of a filled variant of it, the filling having the relative permittivity $\varepsilon_{r}^{(1)}=4$ and the relative permeability $\mu_{r}^{(1)}=1$. The frequency ranges are chosen in both cases such that the ill matching of the waveguide to free space close to the cutoff frequencies to be demonstrated.

In all cases, the waveguide is assumed to be excited by a $T E_{10}$ mode of unit amplitude (in the case of the 2-D analysis, this corresponds to an exciting $E$ and an exciting $H$ polarized $L S E$ mode of unit amplitude).

\section{B. 2-D Analysis of the Rectangular Waveguide}

The computation time required for solving this 2-D electromagnetic problem amounted to $8 \mathrm{~s}$ for the fixed frequency problem and to $50 \mathrm{~s}$ for the spectral analysis one, on a PentiumIV/1.4 GHz personal computer.

The distribution of the electric field inside the waveguide, in the vicinity of the radiating aperture and in the near-field region is illustrated, for the case of the fixed frequency, in Fig. 3. In Fig. 3(a), two phenomena that are associated with the waveguide being operated very close to the cutoff frequency are highlighted:

- the ill-matching of the waveguide, reflected in the reduced amplitude of the field at the aperture;

- the very large wavelength of the standing wave inside the waveguide, reflected in an almost flat distribution of the field inside it.

As for the $H$ polarization analysis, Fig. 3(b) illustrates very well the singular behavior of the field in the vicinity of the edges of the waveguide.

The results concerning the spectral analysis are illustrated by examining the frequency dependence of the antenna's gain

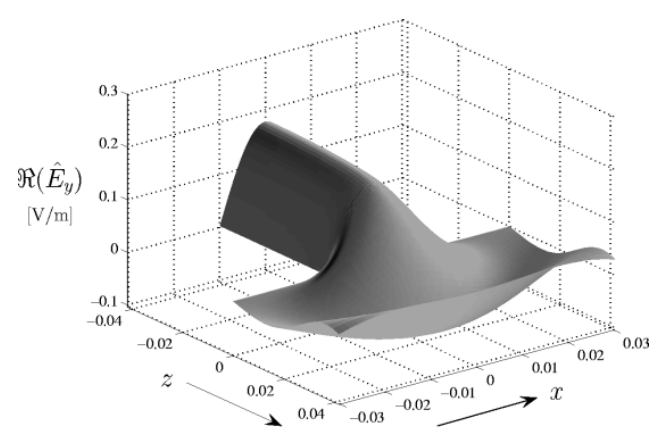

a

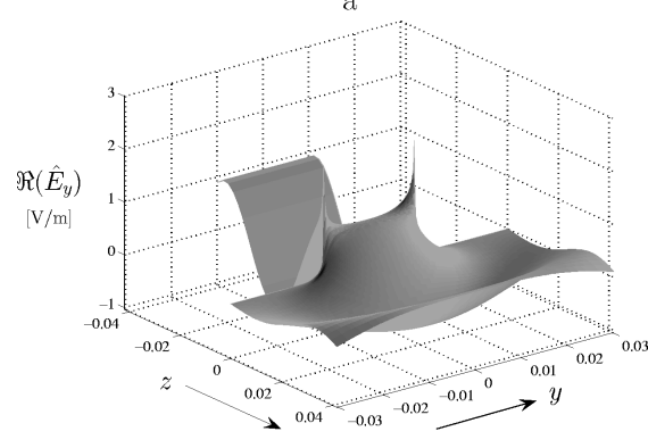

b

Fig. 3. A 2-D analysis: distribution of $\Re\left(\hat{E}_{y}\right)$ in the vicinity of the radiating aperture: (a) $E$ polarization and (b) $H$ polarization.

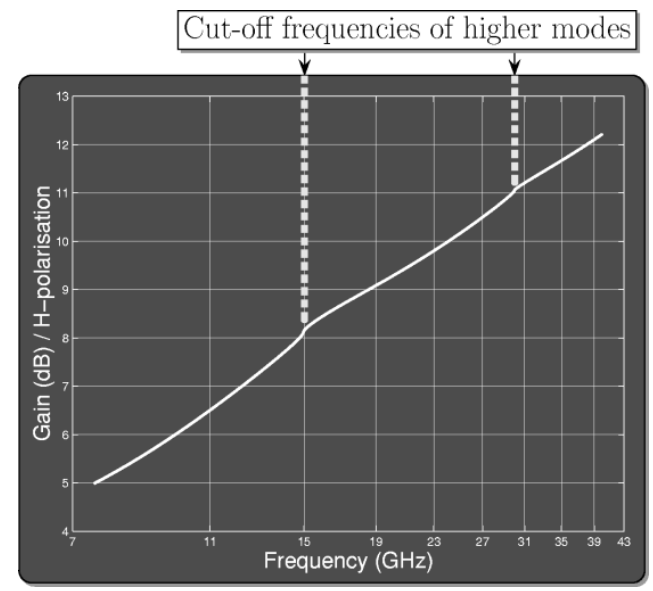

Fig. 4. A 2-D analysis: frequency dependence of the antenna's gain.

(see Fig. 4). Note that the transition to the over-moded operation is clearly visible in this figure.

\section{3-D Analysis of the Rectangular Waveguide}

The computation time required for solving this 3-D electromagnetic problem amounted to approximately $20 \mathrm{~min}$ on the same PentiumIV/1.4-GHz personal computer. The relatively long run-time is determined, mainly, by the limitations arising from the use of the Matlab environment. It is expected that transferring the computational engine into a directly executable program will drastically increase the computational speed.

The single-frequency analysis of the WR90 rectangular waveguide is intended to illustrate some remarkable features of the field in the vicinity of the radiating aperture. Such features are ignored when, in view of simplifying the exposition of the topic, the distribution of the electromagnetic field at the 


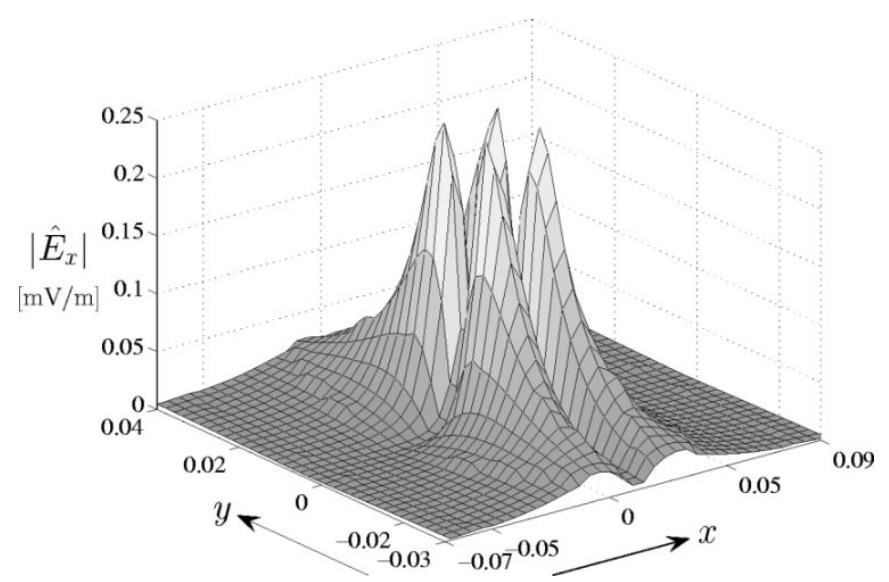

Fig. 5. A 3-D analysis: distribution of $\left|\hat{E}_{x}\right|$ for $\left\{z=7.6 \cdot 10^{-3}\right\}$.

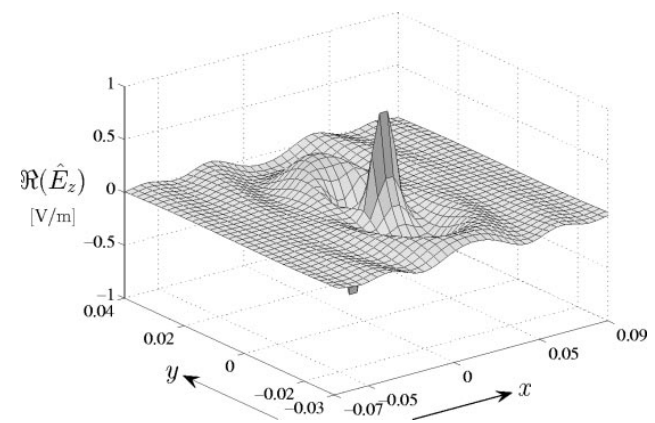

Fig. 6. A 3-D analysis: distribution of $\Re\left(\hat{E}_{z}\right)$ for $\left\{z=2.86 \cdot 10^{-4}\right\}$.

aperture is taken to be elementary (most often, corresponding to a $T E_{10}$ mode). Nevertheless, these features are responsible for the occurrence of (parasitic) effects (for example, the generation of the cross-polarized components of the field) that are of a particular interest in the design of high-performance antennas.

The distribution of the $\left|\hat{E}_{x}\right|$ quantity in the plane $\{z=7.6$. $\left.10^{-3} \approx \lambda_{0} / 4\right\}$ (with $\lambda_{0}$ denoting the wavelength in free space) is represented in Fig. 5. It clearly indicates the fact that the $\hat{E}_{x}$ cross-polarized component concentrates mainly at the corners of the waveguide. This observation is confirmed by practical experience, the cross-polarized radiation pattern being typically measured in a diagonal plane, orthogonal to the plane of the aperture [3].

Another component of the electric field whose behavior is often overlooked in antenna courses is $\hat{E}_{z}$. In Fig. 6 is presented the distribution of the quantity $\Re\left(\hat{E}_{z}\right)$ in the plane $\{z=$ $\left.2.86 \cdot 10^{-4} \approx 0.03 \lambda_{0}\right\}$. The singular behavior of the field in the vicinity of the waveguide's edges is evident. It is emphasized that this component will give the key contribution to the side-lobes level. Since the fundamental mode in the transmission line is transversely electric, the longitudinal component of the electric field can only be due to the higher order modes that

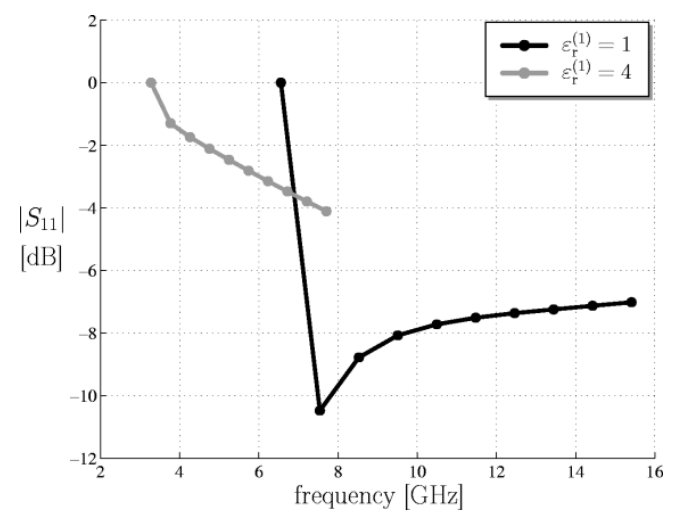

Fig. 7. A 3-D analysis: frequency dependence of the return loss.

are excited at the aperture. Since these modes are all evanescent, $\hat{E}_{z}$ will be rapidly attenuated inside the waveguide. Nevertheless, this component will beget a traveling wave in the free space.

The frequency dependence of the return loss for the fundamental $T E_{10}$ mode on the investigated rectangular waveguide (here, interpreted as a transmission line) constitutes a typical example for a circuit element-type antenna parameter analysis. The examined frequency range was $f=6.6, \ldots, 15.5 \mathrm{GHz}$ in the case of the standard WR90 waveguide and $f=$ $3.5, \ldots, 7.7 \mathrm{GHz}$ in the case of the dielectric-filled variant. The results of this analysis are summarized in Fig. 7. From this figure, the ill matching of the dielectric-filled waveguide over the chosen frequency range can first be observed. A more subtle observation concerns the ill matching of the empty waveguide close to the cutoff frequency of the $T E_{10^{-}}$mode, i.e., $6.574 \mathrm{GHz}$.

\section{CONCLUSION}

A simulation platform for the analysis of the radiating aperture of a (dielectric-filled) waveguide was described. The choices that were made in order to adapt the software tool to the requirements of the educational process were discussed. The capabilities of the software platform were demonstrated by analyzing a number of case studies.

\section{REFERENCES}

[1] F. Thollon and L. Nicolas, "A computer aided education tool for electromagnetic scattering phenomena," IEEE Trans. Magn., vol. 36, pp. $880-883$, July 2000.

[2] G. Conciauro, M. Guglielmi, and R. Sorrentino, Advanced Modal Analysis: CAD Techniques for Waveguide Components and Filters. Chichester, U.K.: Wiley, 2000.

[3] T. S. Bird, "Analysis of mutual coupling in finite arrays of different-sized rectangular waveguides," IEEE Trans. Antennas Propagat., vol. 38, pp. 166-172, Feb. 1990.

[4] I. Lager and A. T. de Hoop, "The mode-matching software package for the dielectric-filled, flanged, parallel-plate, $H$-polarized antennas," IRCTR, Faculty of Information Technology and Systems, Delft Univ. Technology, Scientific Rep. IRCTR-S-001-02, 2002. 